\title{
SAKe: Computationally Designed Modular Protein Building Blocks for Macromolecular Assemblies
}

\author{
Staf M. L. Wouters ${ }^{1}$, David E. Clarke ${ }^{1,2}$, Hiroki Noguchi ${ }^{1}$, Steven De Feyter ${ }^{2}$, Arnout R. D. Voet ${ }^{1}$ \\ ${ }^{1}$ Laboratory of Biomolecular Modeling and Design, Department of Chemistry, \\ KU Leuven, Celestijnenlaan 200G, 3001 Heverlee, Belgium; \\ ${ }^{2}$ Division of Molecular Imaging and Photonics, Department of Chemistry, | \\ KU Leuven, Celestijnenlaan 200F, 3001 Heverlee, Belgium \\ staf.wouters@kuleuven.be
}

\begin{abstract}
Advances in computational protein design have allowed for the development of new proteins with unique properties. Symmetric designer proteins have remarkable stability and can serve as versatile building blocks for the creation of macromolecular assemblies. Here we present the development and structural determination of SAKe: A new symmetric, stable protein building block with modifiable loops. Following the observation of $\mathrm{pH}$ induced 3D self-assembly, we engineered metal binding sites along the protein's internal rotational axis to fabricate 2D surface arrays. Using atomic force microscopy, we demonstrated $\mathrm{Cu}$ (II) dependent on-surface 2D self-assembly. Additionally, using dynamic light scattering and x-ray diffraction, we identified and characterized a SAKe mutant which shows in solution $\mathrm{Zn}$ (II) mediated nanocage formation. This work showcases a stable and highly modifiable SAKe protein scaffold, which holds promise as a building block for the creation of multi-functional macromolecular materials.
\end{abstract}

Keywords: assembly, computational, layers, cages, protein design 